\title{
Molecular Gas in Galaxies at all Redshifts
}

\author{
Françoise Combes \\ Observatoire de Paris, LERMA and CNRS, 61 Av. de l'Observatoire, F-75014 Paris, France \\ email: francoise.combes@obspm.fr
}

\begin{abstract}
I review some recent results about the molecular content of galaxies, obtained essentially from the CO lines, but also dense tracers, or the dust continuum emission. New results have been obtained on molecular cloud physics, and their efficiency to form stars, shedding light on the Kennicutt-Schmidt law as a function of surface density and galaxy type. Large progress has been made on galaxy at moderate and high redshifts, allowing to interprete the star formation history and star formation efficiency as a function of gas content, or galaxy evolution. In massive galaxies, the gas fraction was higher in the past, and galaxy disks were more unstable and more turbulent. ALMA observations will allow the study of more normal galaxies at high z with higher spatial resolution and sensitivity.
\end{abstract}

Keywords. galaxies: general, galaxies: high-redshift, galaxies: evolution, ISM: molecule, galaxies: ISM, galaxies: spiral, galaxies: starburst, galaxies: structure

\section{Nearby galaxies}

A new survey (HERACLES) has been completed with the IRAM receiver array in the $\mathrm{CO}(2-1)$ line, allowing extended maps of nearby galaxies, at 12" resolution (Leroy et al. 2009). The survey contains an atlas of 18 nearby galaxies, observed at multi-wavelengths, and in particular in the HI line, and in the mid infrared by Spitzer. Among the results, it is interesting to note a very good correlation between $\mathrm{CO}$ and $\mathrm{HI}$ kinematics. The excitation of the molecular gas, as traced by the first two rotational lines of $\mathrm{CO}$, is usually low in the disk $(\mathrm{R}=\mathrm{CO}(2-1) / \mathrm{CO}(1-0)=0.6)$ while it is higher in nuclei $(\mathrm{R}=$ $1)$, indicating denser gas. The $\mathrm{CO}$ emission is compatible with optically thick clouds at a kinetic temperature of $\mathrm{T}=10 \mathrm{~K}$.

A more refined view of the star formation law in galaxies has been obtained by Bigiel et al. (2008). The Schmidt-Kennicutt law relating star formation and gas density has a different slope $n$, according to the gas surface density. At high surface density, when the gas is molecular, the gas forms stars at a constant efficiency $(n=1)$, and the time-scale for star formation is $210^{9}$ yrs. While, at low surface density, when the gas is atomic, the slope is much higher $n=2$ or more. At sub-kpc scale, the star formation rate is not strongly correlated with $\mathrm{HI}$ surface density. The transition between $\mathrm{HI}$ and $\mathrm{H}_{2}$ occurs when the surface density is $>9 \mathrm{M}_{\odot} \mathrm{pc}^{-2}$.

In order to better determine the change across the spiral arms of the molecular gas physical properties, Schinnerer et al. (2010) have mapped at interferometric resolution several lines of $\mathrm{CO}$ and its isotopes, together with dense gas tracers, such as HCN and $\mathrm{HCO}^{+}$, in two selected regions across M51 spiral arms. They find no change across the arms, and the GMC population in the spiral arms of M51 is similar to those of the Milky Way, even if the star formation rate is much higher.

On the contrary, the low surface brightness dwarf spiral M33 reveals different conditions than in the Milky Way. In the center, the lower metallicity is the cause of a higher conversion factor, as will be described by J. Braine in this conference (see Gratier 
et al. 2010), while surprisingly the outer molecular complexes show exceptionally bright CO emission, and therefore a lower conversion factor (Bigiel et al. 2010).

The excitation of the molecular gas has also been investigated with the first $3 \mathrm{CO}$ rotational lines, at high resolution with the PdB and SMA interferometers in two NUGAsample galaxies (Boone et al. 2010). At about 100pc-scale resolution, it is possible to distinguish warmer and less dense gas towards the center, may-be heated by the AGN, and colder and denser components in a circumnuclear arc. According to LTE analysis, more then $50 \%$ of the gas is optically thick in both galaxies.

The CO excitation has recently ben estimated even more completely by determining the spectral line energy distribution or SLED in bright starbursts and quasars, with the Herschel Spire FTS instrument. It is possible to obtain the full spectrum at once, although with low spectral and spatial resolution, up to the $\mathrm{CO}(13-12)$ line. In Messier 82, the SLED obtained by Panuzzo et al. (2010) reveals a significant part of the molecular gas at high temperature $\mathrm{T}=500 \mathrm{~K}$, where the $\mathrm{H}_{2}$ lines are the main coolant. The peak of the CO line intensity occurs at the level $J=7$. Extremely high CO line excitation is a clue to the presence of an AGN and its strong X-ray heating (XDR). The star formation region are characterized by $\mathrm{PDR}$, where dust is heated efficiently. Also there is a richer chemistry in XDR $\left(\mathrm{H}_{2} \mathrm{O}, \mathrm{H}_{2} \mathrm{O}+, \mathrm{OH}+..\right)$. Figure 1 reveals the CO SLED of Mrk231, a typical quasar, where the excitation is dominated by an XDR.

For more common lower-energy AGN, the XDR is visible only very close to the nucleus. The ALMA spatial resolution is then required to resolve these regions. A typical example is the Seyfert 2 galaxy NGC 1068, where the XDR dominates the starburst regions only at $\mathrm{r}<70 \mathrm{pc}$. Garcia-Burillo et al. (2010) have mapped SiO, CN with the IRAM interferometer, which are tracers of shocks as well as $\mathrm{CH}_{3} \mathrm{OH}, \mathrm{HNCO}$.

The starbursts in barred galaxies are frequently found in nuclear rings, at Lindblad resonances, such as in NGC1097. The recent observation with Herschel of the dust morphology in this ring by Sandstrom et al. (2010) has revealed a high uniformisation of

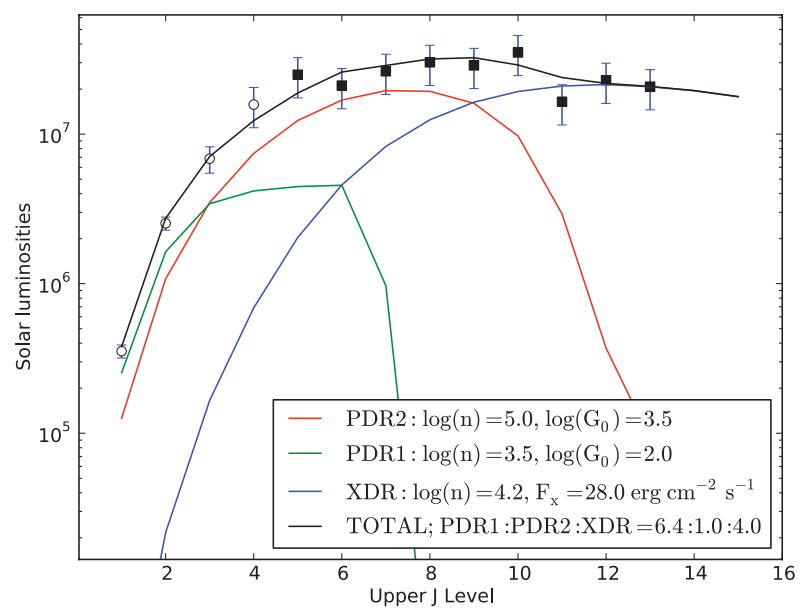

Figure 1. Energy distribution in the various CO lines from Mrk231: the high frequency measurements (filled symbols) have been done with SPIRE on Herschel, while the low frequency ones (open symbols) are measured from the ground. The data can be reproduced by the combination of three models: two model PDR components (red and green lines) and an XDR component (blue line). The sum of these three components is the black line, made to fit the CO measurements. The PDR alone are not sufficient. From van der Werf et al. (2010). 
the dust heating in the ring, suggesting some kind of smoothing, or that the ISRF is a significant source of heating. This smooth structure of the dust contrasts with the clumpy nature of the line emission, in the infrared lines: [OI] 63 $\mu$, [OIII] $88 \mu$, [NII] $122 \mu$, [CII] $158 \mu$ and [NII] $205 \mu$ (Beirao et al. 2010).

Galaxy formation models required strong feedback to limit star formation. This feedback is observed as molecular gas outflow out of starbursting region, such as the prototypical dwarf galaxy M82. Recently, gas outflows have been observed also generated by AGN feedback, in the nearby quasar Mrk 231, with $\mathrm{OH}$ lines and outflow velocities up to $1400 \mathrm{~km} / \mathrm{s}$ (Fischer et al. 2010). In the same quasar, the observation of the $\mathrm{CO}(1-0)$ line with the IRAM interferometer revealed broad wings, with velocities up to $750 \mathrm{~km} / \mathrm{s}$, dragging the molecular gas in strong outflows (Feruglio et al. 2010). According to this interpretation, most of the gas around the nucleus could be depleted in a time-scale of tens of Myrs. In Mrk 231, Gonzalez-Alfonso et al. (2010) have reported about emission and absorption lines of $\mathrm{H}_{2} \mathrm{O}$, which is abundant due to shocks, or XDR chemistry, and evaporation of ice from grains. A clear example of outflows has also been seen in $4 \mathrm{C} 31.04$ (Garcia-Burillo et al. 2007). The $\mathrm{HCO}^{+}$profile is very wide, broader than $1000 \mathrm{~km} / \mathrm{s}$. There is both emission and deep absorption in the blue-side, and an interferometer map has been able to map both, according to its spatial coincidence with the resolved jet in radio continuum. Another striking example, is the radio source 3C293, observed in HI absorption with a width of $1400 \mathrm{~km} / \mathrm{s}$ (Morganti et al. 2003). The absorption is blueshifted, indicating an outflow. Garcia-Burillo et al. (2010) have mapped the source in $\mathrm{CO}$ and $\mathrm{HCO}^{+}$, where a deep absorption is also detected.

The strongest evidence of AGN feedback until now has been seen in the center of cool core clusters. Cold molecular gas has been detected in the CO lines, associated to the cooling flow in Perseus (Salome et al. 2006). Recently, the cooling lines OI and CII have been mapped with PACS and SPIRE in some cooling flows (Edge et al. 2010, Mittal et al. in prep). They were seen with the same morphology, and when possible with the same kinematics than for the CO lines. They appear to come from the same gas, cooling through different phases, showing no rotation, but in(-out)flows.

A strong result from the first Science with Herschel is the evidence of a cold dust component in dwarfs and the outer parts of spiral galaxies (Grossi et al. 2010). This has been confirmed with Planck, and also ground based bolometer, such as LABOCA. In dwarfs, the CII/CO ratio is very high, due to low-metallicity effects (Cormier et al. 2010): the high UV environment, due to the lack of dust, provides large-scale photodissociation of the molecular gas. However, the SED of dust emission reveals and overabundance of cold dust, or a flat opacity law $\beta<1.5$ in low-Z systems (Boselli et al. 2010). This is the case in the LMC (Meixner et al. 2010), and also M33 (Quintana-Lacaci et al. in prep). A 2-component grey body fit with $\beta=2$ indicates a very cold component at $5.7 \mathrm{~K}, 15$ times more massive than the $21 \mathrm{~K}$ component. This excess is clearly seen in the low- $\mathrm{Z}$ dwarf NGC 1705 (O'Halloran et al. 2010).

\section{Galaxies at high redshift}

In the last decade, a large number of $\mathrm{CO}$ detections have occured at high redshift, and surprising is the very good correlation between the FIR and CO fluxes, even for quasars where the AGN contribution to the FIR might be significant (Iono et al. 2009). In the early universe, about a dozain of objects at $z>4$ have been detected in $\mathrm{CO}$, but they are all lens amplified by a large factor (Wang et al. 2010, Riechers et al. 2010a) The most distant object is the QSO at $\mathrm{z}=6.4$ (Fan et al. 2003). The mass of dust is $10^{8} \mathrm{M}_{\odot}$ (Bertoldi et al. 2003), and its black hole mass $1.510^{9} \mathrm{M}_{\odot}$ (Willot et al. 2003). 
The CII line has been detected by Walter et al. (2009), and the data are consistent with a $1 \mathrm{kpc}$-scale starburst, of SF density $1000 \mathrm{M}_{\odot} / \mathrm{yr} / \mathrm{kpc}^{2}$. Surprisingly HCN has not been detected in this source, although HCN appears better correlated to star formation than CO (Gao \& Solomon 2004).

$\mathrm{HCN}$ as a high density tracer has been detected at high $\mathrm{z}$ tracing $\mathrm{nH}_{2} \sim 10^{5} \mathrm{~cm}^{-3}$. One of the strong detections is the Cloverleaf quasar at $\mathrm{z}=2.56$, dominated by a starburst. $\mathrm{CO}, \mathrm{HCN}, \mathrm{HCO}^{+}$trace the warm and dense gas forming stars (Riechers et al. 2010b). In $\mathrm{APM} 08279+5255$, at $\mathrm{z}=3.91$, the excitation is radiative in addition to collisional, under the influence of the massive black hole (Riechers et al. 2010c). In some of the compact star forming regions, the SFR is so high that the gas surface density might be limited by the radiation. In other words, we are seing Eddington limited star formation, at about $\mathrm{SFR}=500-1000 \mathrm{M}_{\odot} / \mathrm{yr} / \mathrm{kpc}^{2}$. The column density is in average $\mathrm{NH}_{2} \sim 10^{24} \mathrm{~cm}^{-2}, 100$ times larger than in Giant Molecular Clouds (Riechers et al. 2009a).

It is very difficult to detect the molecular component of more "normal" objects, with a moderate star formation rate. This is only done with the help of gravitational lenses: the "cosmic eye" corresponds to a Lyman-break galaxy (LBG) at $\mathrm{z}=3.07$ detected in $\mathrm{CO}(3-2)$ with the IRAM interferometer, with a magnification factor of 28 . Its SFR is 60 $\mathrm{M}_{\odot} / \mathrm{yr}$, and its molecular content $\mathrm{MH}_{2}=2.410^{9} \mathrm{M}_{\odot}$, implying a starburst time-scale of 40Myr (Coppin et al. 2007).

Another category of sources frequently detected in molecules are the SMG (Submillimeter Galaxies), discovered through their dust continuum in blind surveys with bolometers. From this dust continuum, redshifted in the millimeter range, it is possible to deduce their rest-FIR flux, which is an indicator of star formation. The molecular content is derived from the CO luminosity, after applying a conversion factor adapted for ultraluminous galaxies (ULIRG). Their star formation efficiency, as traced by the ratio between the FIR and CO luminosities is strongly increasing with redshift (Greve et al. 2005). In

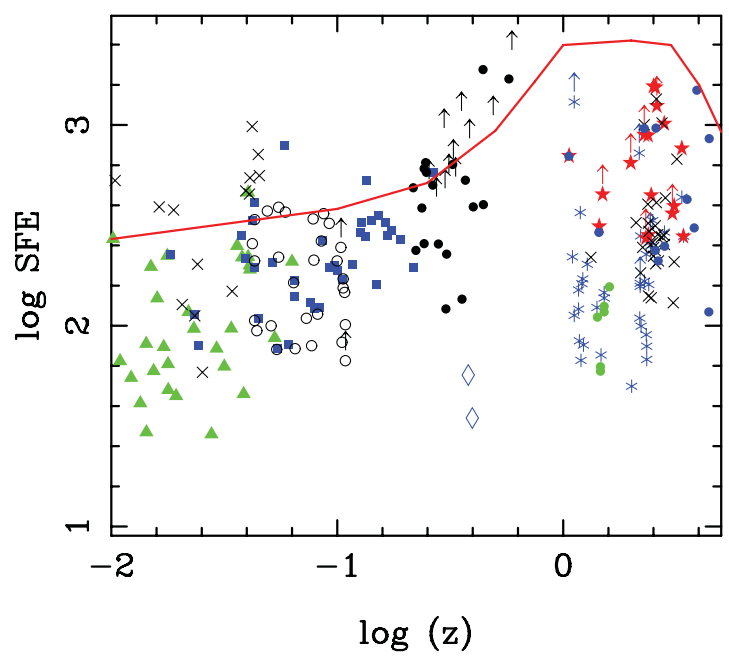

Figure 2. Evolution with $\mathrm{z}$ of the Star Formation Efficiency (SFE) defined by the ratio between far-infrared luminosity and molecular gas mass $\left(\mathrm{L}_{\mathrm{FIR}} / \mathrm{M}\left(\mathrm{H}_{2}\right)\right)$, for the sources detected between $0.2<\mathrm{z}<0.6$ (black full dots, and upward-arrows), compared with other detections in the literature (cf Combes et al. 2010). The red curve is a schematic line summarizing the cosmic star formation history, from the compilation by Hopkins \& Beacom (2006), implemented with the GRB data by Kistler et al. (2009), and the optical data from Bouwens et al. (2008). 
average, their star formation rate is $700 \mathrm{M}_{\odot} / \mathrm{yr}$, with a starburst time-scale of 40-200 Myr. SMG appear more efficient to form stars than ULIRG. This could be due to galaxy mergers, with objects not yet stabilized by bulges, the latter accumulating mass later.

Through selection effects, and because of the negative K-correction in the dust continuum emission at high redshift, objects at $z>1$ have been much more studied than those at intermediate redshift. To fill the gap, we have undertaken a search for $\mathrm{CO}$ emission at $0.2<z<1$, selecting the brightest ULIRGs at these redshifts (Combes et al. 2010). One of the first results between $0.2<z<0.6$ is the increase of the gas content with $z$. The detection rate is about $60 \%$, and the average $\mathrm{H}_{2}$ mass is $1.610^{10} \mathrm{M}_{\odot}$, taking the low CO-to- $\mathrm{H}_{2}$ conversion factor assumed for ULIRGs. The maximum amount of gas available for a single galaxy is quickly increasing as a function of redshift. The star formation efficiency (SFE), traced by the ratio of FIR to CO luminosities, is found to be significantly higher, by a factor 3, than for local ULIRGs, and are comparable to high redshift ones (Figure 2). The increase of SFE with $\mathrm{z}$ follows the increase of star formation.

ULIRG are often detected in the pure rotational lines of $\mathrm{H}_{2}$ (e.g. by Spitzer). However, recently Zakamska (2010) has shown that most of the $\mathrm{H}_{2}$ emission comes from outside the starburst region. Indeed, the $\mathrm{H}_{2}$ lines do not suffer dust obscuration, like the other tracers of SF, or PAH. $\mathrm{H}_{2}$ emission could be due to schocks outside SF regions, triggered by galaxy interactions. $\mathrm{H}_{2}$ then accelerates the cooling and collapse of the gas.

ULIRG are not all of high SF efficiency, there exists a category of luminous galaxies with low efficiency of star formation, where the molecular content is more abundant, and spatially extended. Daddi et al. (2008) have optically selected BzK galaxies, and detected in them much more CO emission than expected. They are massive galaxies, with $\mathrm{CO}$ sizes up to $10 \mathrm{kpc}$, with FIR luminosities of $10^{12} \mathrm{~L}_{\odot}$. They have a normal SFE, with a high gas content, $\mathrm{M}\left(\mathrm{H}_{2}\right) \sim 210^{10} \mathrm{M}_{\odot}$, so that the gas consumption time-scale is $\sim 2$ Gyr, like the Milky Way. Their spatial extent explains why their CO-line excitation is low, compared to nuclear starbursts. In BzK-21000, for instance, at $\mathrm{z}=1.52$, only weak $\mathrm{CO}(3-2)$ has been detected (Dannerbauer et al. 2009), and the CO-SLED is peaking at $J=3$ (as in the MW). This leads to the choice of the standard CO-to- $\mathrm{H}_{2}$ conversion factor, 4.5 times higher than for ULIRGS. Due to selection effects, it is possible that SMG studies have missed a much larger population of gas-rich galaxies at high redshift.

A recent survey of massive star forming galaxies, selected optically to have comparable ranges of mass and $\mathrm{SFR}$ in the AEGIS and BX/BM surveys at $\mathrm{z} \sim 1.2$ and 2.3 was reported by Tacconi et al. (2010). About 20 galaxies were observed at IRAM-PdBI, with a high detection rate $>75 \%$, in these "normal" massive $\left(\mathrm{M}_{*} \sim\right.$ a few $\left.10^{11} \mathrm{M}_{\odot}\right)$ Star Forming Galaxies (SFG). The gas fraction appears to increase with redshift, being in average $34 \%$ and $44 \%$ at $\mathrm{z}=1.2$ and 2.3 respectively. A typical map is shown in Figure 3, revealing a rotating disk, perturbed by massive clumps. The high gas content, with respect to local spirals, implies more gravitational instabilities favoring the formation of clumps. The survey prolongs the correlation between SFR versus stellar mass and redshift. If the star formation rate was higher in the past, it is partly due to the higher gas fraction in galaxies, and also a little bit from a higher SF efficiency with z. Given the short gas consumption time-scale in these objects, galaxies must continuously accrete mass to explain the average gas fractions as a function of time.

It is interesting to note the parallel mass assembly in galaxies and black holes, across time. Many of the objects detected in $\mathrm{CO}$ at high z are AGN. Is there an influence of AGN feedback on CO emission? A good example is the lensed quasar APM08279+5255 at $\mathrm{z}=3.9$ (amplification factor $\sim 50$ ). This object is one of the brightest in the sky, and has been observed with $\mathrm{mm}$ and $\mathrm{cm}$ telescopes. Lines from $\mathrm{CO}(1-0)$ to $\mathrm{CO}(11-$ 10) have been detected. Recent 0.3" resolution $\mathrm{CO}(1-0)$ mapping with VLA (Riechers 
et al. 2009b) reveals that the emission is compact, and not extended, as previously believed. CO emission is co-spatial with optical/NIR, in a circumnuclear disk of 500pc around the black hole. The gas mass is $1.310^{11} \mathrm{M}_{\odot}$, while the black hole mass is $\mathrm{M}_{B H}$ $=2.310^{10} \mathrm{M}_{\odot}$. There is no hint of the influence of the AGN feedback. In this object, as many others at high $\mathrm{z}$, the bulge mass derived from the CO line-width, is 10 times less massive than the value expected from the $\mathrm{M}_{B H}-\sigma$ relation.

More statistically, Wang et al. (2010) have displayed the quasars studied at $\mathrm{z} \sim 6$, and also between $1.4<z<5$, on the $\mathrm{M}_{B H^{-}} \sigma$ diagram, revealing an order of magnitude higher $\mathrm{M}_{B H}$ than expected. However, some caveats must be taken into account, such as the unknown inclination, or other selection biases. For instance, it is easier to detect the CO line in low-inclination objects, with a narrower line-width. ALMA will resolve the morphology of the $\mathrm{CO}$ emission, and determine actual inclinations.

An important progress has been made recently, thanks to the enhanced bandwidth of millimeter receivers. It is now possible to search for redshift with only the CO lines. For objects with $\mathrm{z}>2.2$, there is always a $\mathrm{CO}$ line in the $3 \mathrm{~mm}$ atmospheric band of $80-116 \mathrm{Ghz}$, since the spacing between lines decreases as $(1+\mathrm{z})^{-1}$. When two lines are detected, it is possible to deduce $\mathrm{z}$, since the frequency difference between 2 lines depend on $J$. Weiss et al. (2009) have used this redshift machine to determine the redshift of SMMJ14009+0252. With the same techniques, Negrello et al. (2010) and Lupu et al. (2010) have determined the redshift of lensed SMG discovered behind galaxy clusters, in the Herschel H-ATLAS survey. At $500 \mu \mathrm{m}$ wavelength, most sources with flux above
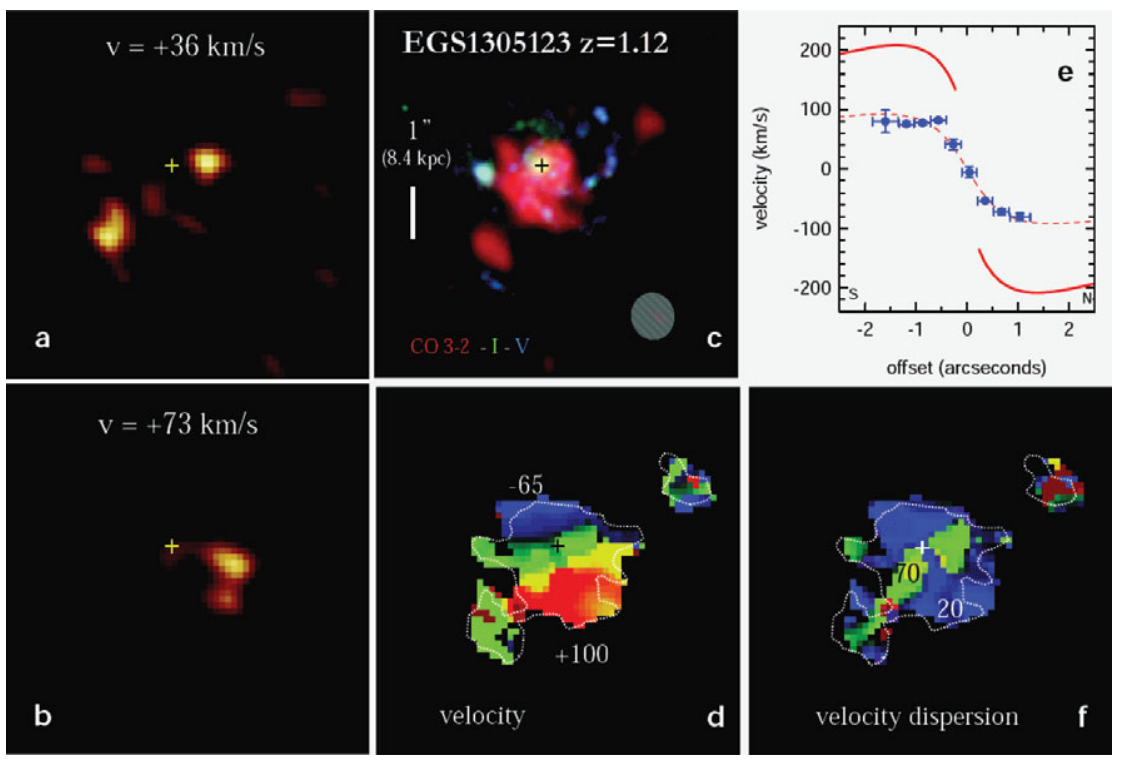

Figure 3. PdB CO(3-2) map at 0.6" x0.7" resolution, of the $\mathrm{z}=1.12$ AEGIS galaxy EGS130512. a \& b: two $9 \mathrm{~km} / \mathrm{s}$ channel maps at +36 and $+72 \mathrm{~km} / \mathrm{s}$, showing the massive molecular clumps, of typical gas masses $\sim 5 \times 10^{9} \mathrm{M}_{\odot}$, intrinsic radii $<1-2 \mathrm{kpc}$, gas surface densities $300-700 \mathrm{M}_{\odot} . \mathrm{pc}^{-2}$ and velocity dispersions $\sim 19 \mathrm{~km} / \mathrm{s}$. c: CO integrated line emission (red), I-band (green) and V-band (blue) HST ACS images of the source. $\mathbf{d} \&$ f: peak velocity and velocity dispersion maps of the $\mathrm{CO}$ emission. e: peak $\mathrm{CO}$ velocity along the major axis $\left(\mathrm{PA}=16^{\circ}\right)$. The best fitting exponential disk model with radial scale length $\mathrm{Rd}=0.77^{\prime \prime}$ and dynamical mass of $2 \times 10^{11} \mathrm{M}_{\odot}$, for an adopted inclination of $27^{\circ}$ is shown as a dotted red curve. The thick red curve is the deprojected rotation curve. 
$100 \mathrm{mJy}$ are lensed SMG, or nearby spirals. In the Science Demonstration Phase, 11 sources were detected in 14 square degrees, and 6 were nearby galaxies. The remaining 5 were indeed lensed SMG, and 4 of them were detected in CO. Observations in the NIR domain give the nature of the lenses. Extrapolating over the 550 square degrees of the survey, more than 100 lensed SMG will be discovered.

Using also the redshift machine at the IRAM-30m, Lestrade et al. (2010) have recently discovered the brightest SMGs in the Northern hemisphere, quite serendipitously. A source of $30 \mathrm{mJy}$ at $1.2 \mathrm{~mm}$ with MAMBO was found, while searching for a debris-disk, but without any local molecular cloud. The redshift search with EMIR at IRAM-30m revealed the $\mathrm{CO}(6-5)$ and (4-3) lines, together with the 2 CI lines, and $\mathrm{CO}(7-6)$ in MM18423+5938, which is likely a lensed ULIRG galaxy, at $\mathrm{z}=3.93$ (see Figure 4). Another lensed star forming galaxy (magnification factor of 32) was discovered at $\mathrm{z}=2.33$ by Swinbank et al. (2010). The size dilation due to the lens allows to resolve the star forming regions at $\mathrm{z}$ $=2.33$. They are 100 times more luminous at a given size than Giant Molecular Clouds in the Milky Way.

\section{Perspectives with ALMA}

The early science of ALMA will begin in 2011. In the near future, ALMA will bring a breakthrough in our knowledge of the molecular content of galaxies. First in continuum, deep fields will give the source count $\mathrm{N}(\mathrm{S})$ to much lower fluxes, and follow-up in the lines will precise the star formation history of the universe. CO lines will be intensively observed at high redshift, and the gas fraction determined for "normal" systems. This will allow to derive the efficiency of star formation as a function of redshift, the gas kinematics, dynamical masses, and clues to the various processes of galaxy formation.

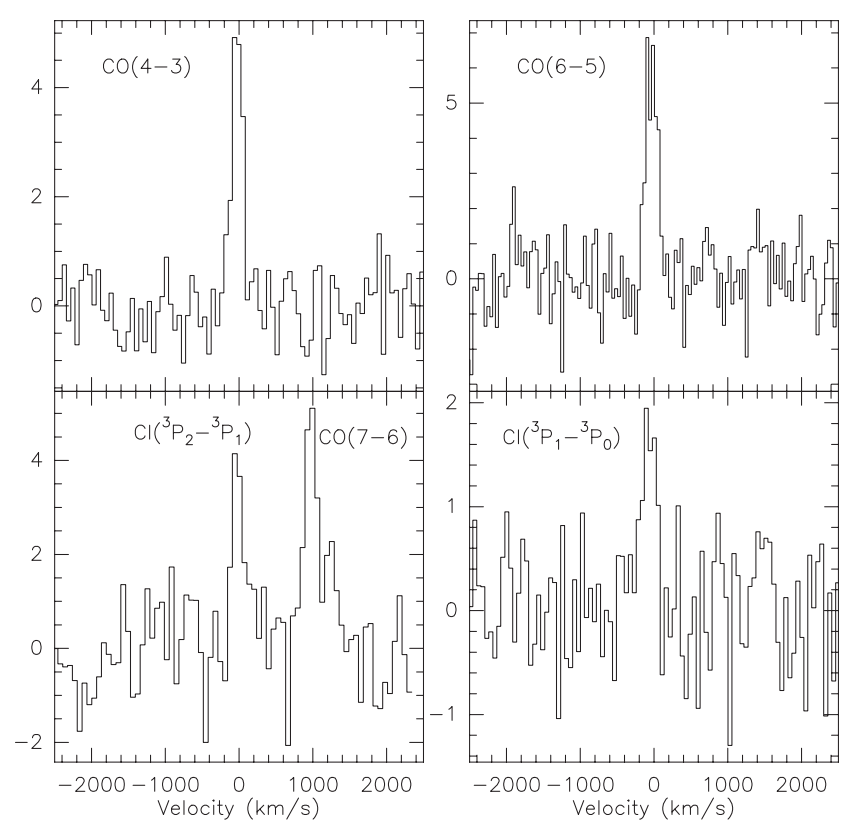

Figure 4. The three CO rotational lines detected, and the two $\mathrm{CI}$ lines, towards the brigtest SMG in the North, MM18423+5938, by Lestrade et al. (2010). The vertical scale is $\mathrm{T}_{m b}$ in $\mathrm{mK}$. 


\section{References}

Beirao, P., Armus, L., Appleton, P. N. et al.: 2010, A\&A 518, L60

Bertoldi, F., Carilli, C. L., Cox, P. et al.: 2003, A\&A 406, L55

Bigiel, F., Leroy, A., Walter, F. et al.: 2008 AJ 136, 2846

Bigiel, F., Bolatto, A. D., Leroy, A. K. et al.: 2010, ApJ 725, 1159

Boone, F., Garcia-Burillo, S., Combes, F. et al.: 2011, A\& A 525, A18

Boselli, A., Ciesla, L., Buat, V. et al.: 2010, A\& $A$ 518, L61

Bouwens, R. J., Illingworth, G. D., Franx, M., \& Ford, H.: 2008, ApJ 686, 230

Combes, F., Garcia-Burillo, S., Braine, J. et al.: 2010, A\&A sub (arXiv1009.2040)

Coppin, K. E. K., Swinbank, A. M., Neri, R. et al.: 2007, ApJ 665, 936

Cormier, D., Madden, S. C., Hony, S. et al.: 2010, A\& $A$ 518, L57

Daddi, E., Dannerbauer, H., Elbaz, D. et al:: 2008, ApJ 673, L21

Dannerbauer, H., Daddi, E., Riechers, D. A. et al.: 2009, ApJ 698, L178

Edge, A. C., Oonk, J. B. R., Mittal, R. et al. 2010, A\&3A 518, L46 and L47

Fan, X., Strauss, M. A., Schneider, D. P. et al.: 2003, AJ 125, 1649

Feruglio, C., Maiolino, R., Piconcelli, E. et al.: 2010, A\&A 518, L155

Fischer, J., Sturm, E., Gonzalez-Alfonso, E. et al.: 2010, A\&A 518, L41

Gao, Y. \& Solomon, P. M.: 2004, ApJS 152, 63

Garcia-Burillo, S., Combes, F., Neri, R. et al.: 2007, A\& A 468, L71

Garcia-Burillo, S., Usero, A., Fuente, A. et al.: 2010 A\&A 419 , A2

Gonzalez-Alfonso, E., Fischer, J., Isaak, K. et al.: 2010, A\& $A$ 518, L43

Gratier, P., Braine, J., Rodriguez-Fernandez, N. J. et al.: 2010, A\& A 522, A3

Greve, T. R., Bertoldi, F., Smail, I. et al.: 2005, MNRAS, 359, 1165

Grossi, M., Hunt, L. K., Madden, S. et al.: 2010, A\&A 518, L52

Hopkins, A. M. \& Beacom J. F.: 2006, ApJ, 651, 142

Iono, D., Wilson, C. D., Yun, M. S. et al. 2009, ApJ 695, 1537

Kistler, M. D., Yüksel, H., Beacom, J. F. et al.: 2009, ApJ 705, L104

Lupu, R. E., Scott, K. S., Aguirre, J. E. et al.: 2010, ApJ in press, arXiv1009.5983

Leroy, A., Walter, F., Bigiel, F. et al.: 2009 AJ 137, 4670

Lestrade, J-F., Combes, F., Salome, P. et al. 2010: A\&A 522, L4

Meixner, M., Galliano, F., Hony, S. et al. 2010: A\& $A$ 518, L71

Morganti, R., Oosterloo, T. A., Emonts, B. H. C. et al. 2003, ApJ 593, L69

Negrello, M., Hopwood, R., De Zotti, G. et al.: 2010, Science 330, 800

O'Halloran, B., Galametz, M., Madden, S. C. et al.: 2010, A $₫ A$ 518, L58

Panuzzo, P., Rangwala, N., Rykala, A. et al.: 2010, A\&A 518, L37

Riechers, D A., Capak, P. L., \& Carilli, C. L.: 2010a ApJ 720, L131

Riechers, D A., Walter F., Carilli, C. L.: 2010b ApJ 726, 50

Riechers, D. A., Weiss, A., Walter, F., \& Wagg, J.: 2010c ApJ 725, 1032

Riechers, D. A., Walter, F., Bertoldi, F. et al. : 2009a, ApJ 703, 1338

Riechers, D. A., Walter, F., Carilli C., \& Lewis G.: 2009b, ApJ 690, 463

Salomé, P., Combes, F., Edge, A. C. et al.: 2006, A $\& A$ 454, 437

Sandstrom K., Krause, O., Linz, H. et al.: 2010, A\&A 518, L59

Schinnerer, E., Weiss A., Aalto, S., \& Scoville, N. Z.: 2010, ApJ 719, 1588

Swinbank, A. M., Smail, I., Longmore, S. et al.: 2010, Nature, 464, 733

Tacconi L. J., Genzel R., Neri R. et al.: 2010, Nature 463, 78

van der Werf, P. P., Isaak, K. G., Meijerink, R. et al.: 2010, A\&A, 518, L42

Walter, F., Riechers, D., Cox, P. et al.: 2009, Nature, 457, 699

Wang, R., Carilli, C. L., Neri, R. et al.: 2010, ApJ 714, 699

Weiss, A., Downes, D., Walter, F., \& Henkel, 2007, ASPC 375, 25

Willott, C. J., McLure, R. J., Jarvis, M. J. et al.: 2003, ApJ 587, L15

Zakamska, N.: 2010 Nature 465, 60 